\title{
IDENTIFICANDO OS USOS TERAPÊUTICOS DA Carapa guianensis
}

Osvaldo Campos dos Santos Nonato ${ }^{1}$, Susan Christina Braga Domingos ${ }^{2}$, Soraia Figueiredo de Souza ${ }^{3}$, Sara Lucena de Amorim ${ }^{3}$, Luciana dos Santos Medeiros ${ }^{3}$

'Discente do curso de Ciências Biológicas da Universidade Federal do Acre/UFAC, Rio Branco, AC.

${ }^{2}$ Mestranda pelo Programa de Pós-Graduação em Sanidade e Produção Animal Sustentável na Amazônia Ocidental/UFAC, Rio Branco, AC.

${ }^{3}$ Professoras da Universidade Federal do Acre/UFAC, Rio Branco, AC. E-mail: lusmedeiros@yahoo.com.br

Recebido em: 22/09/2018 - Aprovado em: 23/11/2018 - Publicado em: 03/12/2018 DOI: 10.18677/EnciBio_2018B86

\section{RESUMO}

A andiroba é utilizada por populações residentes na região Amazônica com uma grande diversidade de uso medicinal. O objetivo deste trabalho consistiu em uma investigação sobre a etnofarmacologia da Carapa guianensis em bases de dados indexadas analisando os usos e manejos realizados. Foram consultados artigos indexados em bases de dados da Internet (PubMed, LILACS, SciELO e Periódicos Capes), entre julho de 2017 e junho de 2018. Considerando o termo de busca "Carapa guianensis", nenhum filtro, como idioma ou intervalo de datas, foi aplicado à pesquisa. A contagem múltipla (duplicação) das publicações foi rastreada e excluída. Um total de 46 artigos indexados, variando entre os anos de 2005 a 2018, que se referiam a $C$. guianensis, foram encontrados em todas as bases de dados. A maioria $(89 \%)$ dos trabalhos publicados foram desenvolvidos na região Neotropical. Este resultado pode ser decorrente da disponibilidade de extratos da planta, normalmente encontrados em países tropicais. Em uma parcela dos 46 $(100 \%)$ trabalhos publicados foi demonstrado que a andiroba pode ser utilizada como insetífugo $(17,40 \%)$, carrapaticida $(6,52 \%)$, anti-inflamatório $(8,70 \%)$, antiparasitário (8,70\%), antimicrobiano $(4,34 \%)$ e antialérgico $(4,34 \%)$. Os estudos de $C$. guianensis revelam que a planta possui fatores fitoquímicos eficientes para 0 seu uso terapêutico. Estes constituintes, identificados nos estudos, são eficazes principalmente como insetífugo, carrapaticida e anti-inflamatório.

PALAVRAS-CHAVE: andiroba, fitoquímicos, usos terapêuticos

\section{IDENTIFYING THERAPEUTIC USES OF Carapa guianensis}

\section{ABSTRACT}

Andiroba is used by populations on the Amazon region with a great diversity of medicinal use. The objective of this study was to investigation the ethnopharmacology of Carapa guianensis on the uses and management, considering indexed databases. We searched for indexed articles in Internet databases (PubMed, LILACS, SciELO and Periodicals Capes) between July 2017 and June 2018. Considering the search term "Carapa guianensis", no filter, such as language or date range, was applied to the research. Multiple counting (duplication) of publications has been crawled and deleted. A total of 46 indexed articles, ranging from 2005 to 2018, referring to $C$. guianensis, were found in all databases. The majority $(89 \%)$ of the published works were developed in the Neotropical region. This result may be due to the availability of extracts from the plant, usually found in tropical countries. In 46 
(100\%) of published studies, it was demonstrated that andiroba can be used as insecticide $(17,40 \%)$, against ticks $(6,52 \%)$, as anti-inflammatory $(8,70 \%)$, as antiparasitic (8,70\%), as antimicrobial (4,34\%) and as antiallergic (4,34\%). The studies of $C$. guianensis reveal that this plant has efficient phytochemical factors for therapeutic use. These constituents, identified in the studies, are effective mainly as insecticide, against ticks and as anti-inflammatory.

KEYWORDS: andiroba, phytochemicals, therapeutic uses.

\section{INTRODUÇÃO}

A floresta amazônica é o ecossistema que possui a maior biodiversidade do mundo. Dentre a fauna da floresta amazônica destaca-se às espécies da família Meliaceae. A família Meliaceae é pertencente a ordem Sapindales, onde se destacam cinco gêneros e 120 espécies encontradas em toda a Região Neotropical, (HENRIQUES; PENIDO, 2014). No Brasil são encontradas 16 espécies, dentre estas a $C$. guianensis (SILVA; ALMEIDA, 2014). Tradicionalmente reconhecida por Carapa e Andiroba, que na língua indígena significa "sabor amargo" (SANTOS; PELLICCIOTTI, 2016), a C. guianensis é uma árvore de folhas perenes que cresce a $60 \mathrm{~m}$ de altura (TONINI et al., 2008).

Os efeitos terapêuticos da $C$. guianensis são decorrentes das propriedades fitoquímicas presentes em toda a planta. O principal produto retirado da planta é o óleo, que possui princípios ativos como glicerídeos, ácidos graxos e tetranortriterpenoides (TNTPS) (MECCIA et al., 2013; MOYA et al., 2015). A amargura de $C$. guianensis está relacionada à presença de limonóides ou tetranortriterpenóides, que conferem atividade anti-inflamatória (HENRIQUES; PENIDO, 2014; WANZELER et al., 2018). Recentemente, foram descritos limonóides do tipo phragmalina andirolideos $A, B, C, D, E, F, G, H, I, J, L, K, M, N, O, P, Q, R$, $\mathrm{S}, \mathrm{T}, \mathrm{U}, \mathrm{V}, \mathrm{X}, \mathrm{W}$ e $\mathrm{Y}$, gedunina e Carapanosina $\mathrm{C}$ foram isoladas de $C$. guianensis (SAKAMOTO et al., 2015; HIGUCHI et al., 2017a; HIGUCHI et al., 2017b; INOUE et al., 2018).

O óleo misturado com cinzas e casca de cacau é utilizado como repelente e inseticida (FERRARI et al., 2007; SANTOS et al., 2014; KLAUCK et al., 2015; NARDI et al., 2016; PARREIRA et al., 2018). O óleo ainda é usado contra carrapatos, pulgas, larvas, piolhos, sarnas e picadas de insetos (BARROS et al., 2012; BARROS et al., 2015; ROMA et., 2015; JESUS et al., 2017). O fruto é usado por alguns insetos como meio de oviposição e nutrição das larvas até a forma adulta. Também sendo utilizado para o tratamento de feridas e massagens terapêuticas (ROSA et al., 2013; SILVA et al., 2015; ARAÚJO et al., 2017).

Adicionalmente, a andiroba possui atividade antiviral (PROPHIRO et al., 2012), atividade antibacteriana (SILVA; ALMEIDA, 2014), antiparasitária (MIRANDA JÚNIOR et al., 2012; OLIVEIRA et al., 2018), cicatrizante (NAYAK et al., 2010; BRITO et al., 2013; SANTOS et al., 2013; SOUZA et al., 2017), antioxidante (ARAÚJO-LIMA et al., 2018) e antialérgica (HENRIQUES; PENIDO, 2014). A utilização errônea pode afetar a morfologia de espermatozóides em alguns animais (ARREBOLA et al., 2013a; PAIXÃO et al., 2016), além de promover a formação de anomalias cromossômicas (ARREBOLA et al., 2013b; LEMES et al., 2017).

Logo os efeitos terapêuticos de $C$. guianensis revelam que produtos naturais podem substituir alguns fármacos anti-inflamatórios e antimicrobianos. Considerando as inúmeras aplicações, foi proposta uma investigação etnofarmacológica da $C$. guianensis em bases de dados indexadas, objetivando um estudo da distribuição temporal e espacial dos trabalhos publicados e analisando seus usos. 


\section{Fontes de dados}

\section{MATERIAL E MÉTODOS}

Bases de dados da Internet (PubMed, LILACS, SciELO e Periódicos Capes) foram consultadas entre julho de 2017 e junho de 2018. Os artigos de cada banco de dados bibliográficos foram selecionados usando os termos "Carapa guianensis". Nenhum filtro, como idioma ou intervalo de datas, foi aplicado à pesquisa. A contagem múltipla (duplicação) das publicações foi rastreada e excluída

\section{Seleção de estudo}

Para todos os artigos, o texto completo foi obtido e analisado. O Microsoft Excel foi utilizado para análise de dados e representação gráfica dos resultados. Os artigos foram excluídos se (i) não pertencessem a espécie $C$. guianenses, sendo de outras espécies do gênero Carapa sp.; (ii) os resultados não foram representados por espécies; (iii) revisões de literatura.

\section{Extração de dados}

Em todos os estudos publicados, a seção de materiais e métodos foi cuidadosamente lida e analisada. Independentemente do tamanho e da significância estatística da amostragem, ou mesmo dos protocolos adotados de testes empregados, foi lido na íntegra e analisado todos os artigos. A fim de obter informações de uso a C. guianensis, os trabalhos foram divididos de acordo com a proposta clínica: como anti-inflamatórios, repelentes, cicatrizantes e/ou antimicrobianos.

\section{RESULTADOS E DISCUSSÃO}

Considerando os termos de busca, um total de 46 artigos indexados, variando entre os anos de 2005 a 2018, que se referiam a C. guianensis, foram encontrados em todas as bases de dados. Segundo o primeiro critério de exclusão (não pertencer a espécie $C$. guianenses) não foram excluídos nenhum artigo, pois todos os trabalhos evidenciaram as espécies do gênero Carapa sp. Não houve necessidade de exclusão de revisões bibliográficas, totalizando a permanência de 46 artigos com a temática de C. guianensis. O primeiro artigo citado no estudo encontrado em uma base indexada foi no ano de 2005 (Figura 1).

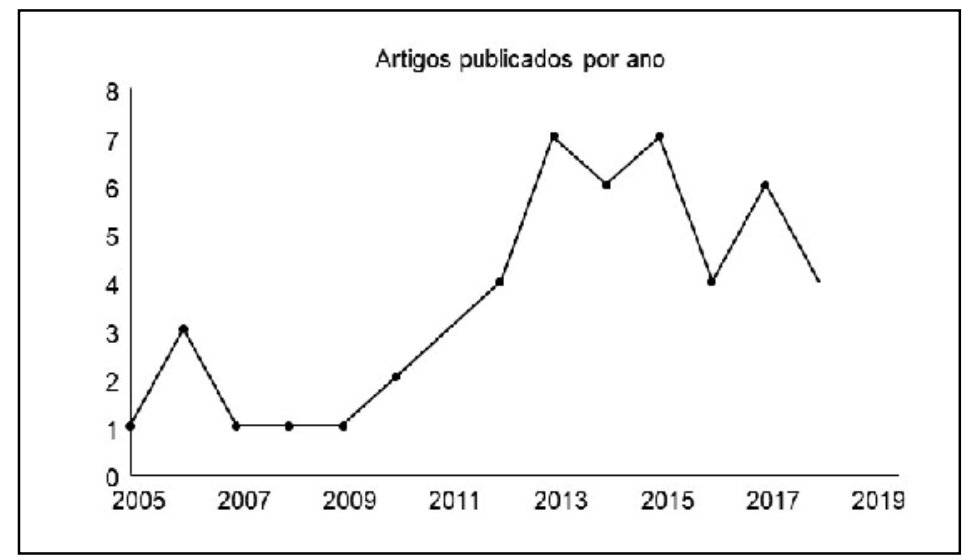

FIGURA 1. Representação quantitativa de artigos relacionados a $C$. guianensis, publicados por ano em revistas indexadas. 
Os anos que apresentaram uma taxa maior de publicações foram 2013 e 2015 com sete artigos que demonstravam as diversas maneiras de aplicação da $C$. guianensis. O maior índice de trabalhos publicados se concentrou entre os anos de 2013 e 2017 com artigos publicados em revistas indexadas. Estes trabalhos abordaram o uso da andiroba como repelente contra insetos, inibidor de vírus e protozoários sanguíneos como o Plasmoduim sp..

A maioria dos trabalhos publicados foram desenvolvidos na região Neotropical (89\%). A figura 2 demonstra o número de publicações por país. O Brasil concentra a maioria dos trabalhos publicados $(78 \%)$ seguido do Japão $(8,6 \%)$. Este resultado pode ser decorrente da disponibilidade de extratos da planta, normalmente encontrados em países tropicais. A andiroba é usada para diversas atividades econômicas pelos moradores da região norte do Brasil, pois eles utilizam de forma artesanal e medicinal (Figura 3).

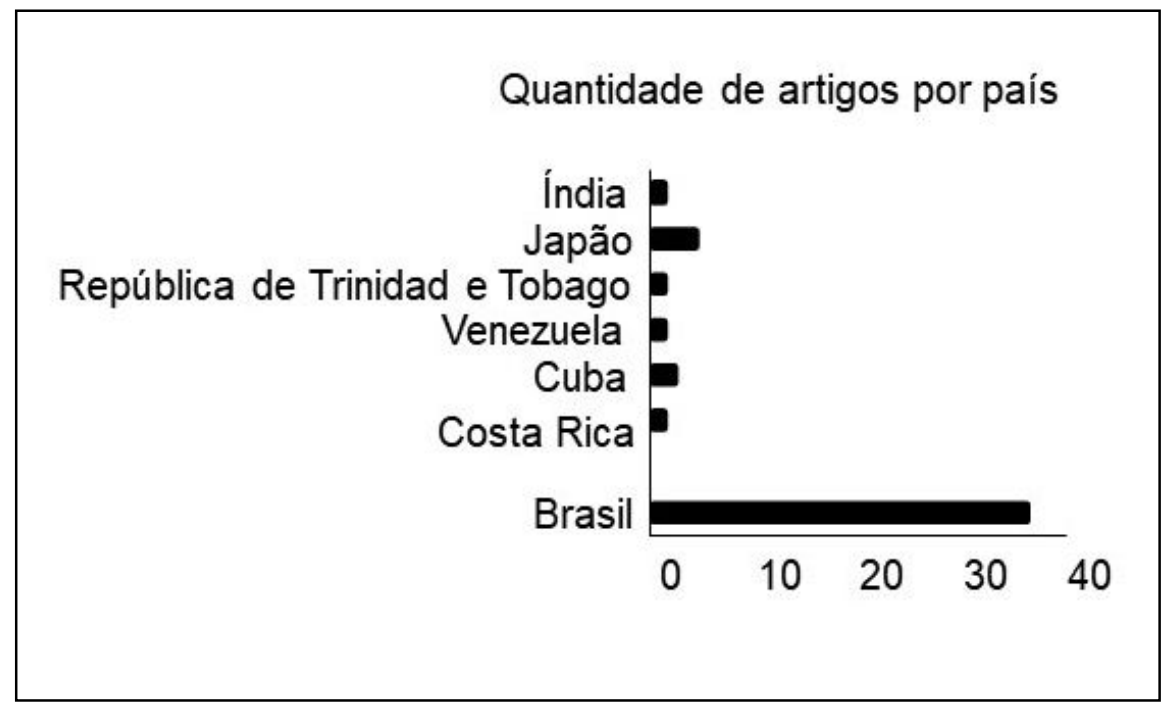

FIGURA 2. Quantificação de artigos relacionados a $C$. guianensis, publicados por país.

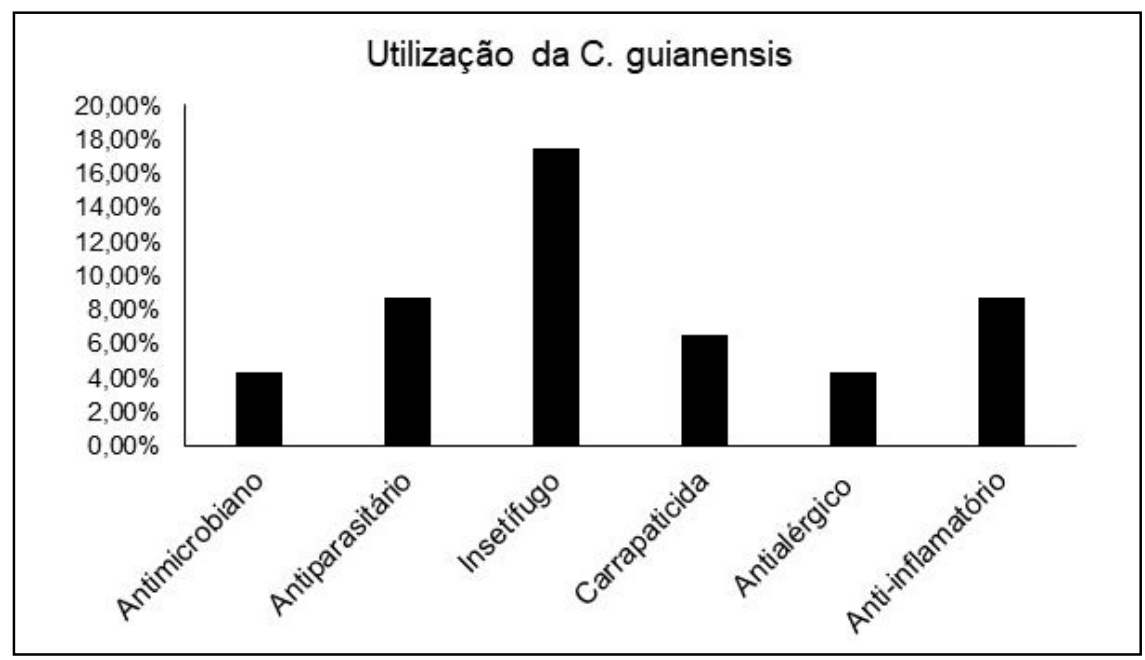

FIGURA 3. Representação quantitativa (\%) dos trabalhos com uso medicinal da $C$. guianensis. 
$\mathrm{Na}$ utilização medicinal é bastante recomendada por apresentar característica anti-inflamatórias, e estudos confirmam essa atividade (PENIDO, et al. 2006). Estudos realizados por Penido e colaboradores (2006), demonstraram que os tetranortriterpenoides têm atuação contra a artrite, doença que afeta as articulações causando dores e rigidez.

Estudos realizados demonstram que a andiroba possui efeitos insetífugos, como repelentes $(17,40 \%)$. Fernandes et al. (2016) demonstraram o uso eficaz do óleo da andiroba no combate com todas as moscas da família Calliphoridae. Em dois estudos realizados por Klauck et al. $(2014 ; 2015)$ foi comprovada a eficiência do óleo da andiroba como repelente contra Musca domestica (moscadoméstica), Chrysomya megacephala (mosca oriental da latrina) e Haematobia irritans (mosca-dos-chifres).

Além de outros estudos destacarem o uso da andiroba como repelente e inseticidas para o Aedes aegypti, mosquito pertencente a ordem Diptera, que é transmissor do vírus da dengue e febre amarela urbana (SILVA et al., 2006; PROPHIRO et al., 2012; TORRES et al., 2014). Nos estudos conduzidos por Farias et al. (2009) e Roma et al. (2013), o óleo da C. guianensis apresentou atividade carrapaticida contra fêmeas ingurgitadas das espécies Anocentor nitens e Rhipicephalus sanguineus. É evidente a utilização de plantas pelas pessoas objetivando a cicatrização de feridas. Em estudo realizado por Santos et al. (2013) a C. guianensis foi utilizada para auxiliar na cicatrização de gastrorrafias em ratos. Os resultados demonstraram que o óleo de andiroba acelerou o processo de cicatrização nesses animais. Foi observado por Souza et al. (2017) uma aceleração na cicatrização de feridas em ratos com diabetes, quando utilizado o óleo da andiroba.

Já em outro estudo feito por Penido et al. (2005), os autores investigaram as propriedades antialérgicas e analgésicas do óleo obtido das sementes de $C$. guianensis que inibiram a exsudação pleural, o edema da pata e da orelha de camundongos sensibilizados, além de possuir propriedades que diminuem a hiperalgesia estimulada por ovalbumina e histamina. Em outro trabalho realizado por Penido et al. (2006) os resultados sugeriram que os TNTPs isolados de $C$. guianensis possuem potenciais farmacóforos atuando na inibição da expressão gênica regulada por NFKB, inibindo a síntese de IL-5 e eotaxina e, portanto, eosinofilia alérgica.

Nos estudos desenvolvidos por Farias et al. (2010) avaliaram nematódeos presentes em caprinos e ovinos, logo se foram presentes os gêneros Haemonchus sp., Oesophagostomum sp., Trichostrongylus sp., Strongyloides sp. Positivos nos animais e por fim evidenciaram a potencialidade do óleo da semente da andiroba no controle desses vermes. Em outro trabalho, Pereira et al. (2014) avaliaram o óleo das sementes de $C$. guianensis na potencialidade antiparasitária e observaram a eficácia in vitrocontra Plasmodium falciparum e adicionalmente in vivo contra Plasmodium berghei em ratos. Em outro estudo desenvolvido por Miranda Júnior et al. (2012) também evidenciaram a eficiência do óleo da semente da andiroba contra o $P$. falciparum.

Adicionalmente foram encontrados poucos trabalhos $(4,34 \%)$ sobre uso da andiroba como antimicrobiano. Em um estudo realizado por Silva e Almeida (2014) demonstrou-se que o óleo de andiroba pode inibir crescimento da Klebsiella pneumoniae, bactéria causadora da pneumonia e infecções hospitalares. Já Meccia et al. (2013), verificaram a inibição do crescimento de Staphylococcus aureus e Enterococcus faecalis, também com o uso do óleo. 


\section{CONCLUSÃO}

Os 46 estudos de $C$. guianensis publicados em revistas indexadas revelaram que a planta possui fatores fitoquímicos eficientes para o uso terapêutico. $\mathrm{O}$ uso do óleo e extrato da planta como anti-inflamatório e antialérgico já é reconhecido pela população da região amazônica. Os conhecimentos são empíricos demonstrados pelas populações regionais e os produtos fitoquímicos da planta podem ser eficazes quando utilizados como antibacterianos e antiparasitários, inibindo os crescimentos de alguns microrganismos pertencentes a esses grupos.

\section{REFERÊNCIAS}

ARAÚJO, A. L.; TEIXEIRA, F. A.; LACERDA, T. F.; FLECHER, M. C.; SOUZA, V. R. $C$. et al. Effects of topical application of pure and ozonized andiroba oil on experimentally induced wounds in horses. Brazilian Journal of Veterinary Research and Animal Science, v. 54, n. 1, p. 66-74, 2017. Disponível em: $<\mathrm{http}: / / \mathrm{dx}$.doi.org/ 10.11606/issn.1678-4456.bjvras.2017.113776>. doi: 10.11606/issn.1678-4456.bjvras.2017.113776

ARAÚJO-LIMA, C. F.; FERNANDES, A. S.; GOMES, E. M.; OLIVEIRA, L. G.; MACEDO, A. F. et al. Antioxidant Activity and Genotoxic Assessment of Crabwood (Andiroba, Carapa guianensis Aublet) Seed Oils. Oxidative medicine and cellular longevity, v. 2018, p. 1-11, 2018. Disponível em: <https://doi.org/10.1155/2018/3246719>. doi: 10.1155/2018/3246719

ARREBOLA, D. F. A.; FERNÁNDEZ, L. A. L; ROCHE, L. D.; LAURENCIO, A. A.; NOVOA, A. V. Potencial genotóxico del extracto oleoso de la semilla de Carapa guianensis Aublet para inducir anomalías en la morfología de la cabeza del espermatozoide. Revista Internacional de Andrología, v.11, n.2, p.54-59. 2013a. Disponível em: <http://dx.doi.org/10.1016/j.androl.2012.12.001>. doi: $10.1016 / \mathrm{j}$. androl.2012.12.001

ARREBOLA, D. F. A.; FERNÁNDEZ, L. A. R.; ROCHE, L. D.; LAURENCIO, A. A.; BOURZACET, C. J. F. I. et al. Evaluación genotóxica del extracto oleoso de la semilla de $C$. guianensis aublet en el ensayo de aberraciones cromosómicas en ratones balb/c. Revista Cubana de Farmacia, v. 47, n. 3, p. 363-367, $2013 b$. Disponível em: < http://scielo.sld.cu/pdf/far/v47n3/far09313.pdf >.

BARROS, C. R. J.; GUEDES, A. C. L.; GUEDES, M. C.; RIBEIRO, G. G.; BARBOSA, E. J. Registro da ocorrência de Hypsipyla ferrealis e Hypsipyla grandella (LEPIDOPTERA: PYRALIDAE) em frutos de andirobeiras (Carapa guianensis, Meliaceae) em Macapá - AP, Brasil. Ciência Florestal, v. 25, n. 3, p. 765-769, 2015. Disponível em: <http://dx.doi.org/10.5902/1980509819679>. doi:10.5902/1980509819679

BARROS, F. N. FARIAS, M. P. O.; TAVARES, J. P. C.; ALVES, L. C.; FAUSTINO, M. A. G. In vitro efficacy of oil from the seed of Carapa guianensis (andiroba) in the control of Felicola subrostratus. Brazilian Journal of Pharmacognosy, v. 22, n. 5, p. 1130-1133, 2012. Disponível em: <http://dx.doi.org/10.1590/S0102695X2012005000047>. doi: 10.1590/S0102-695X2012005000047

BRITO, N. B.; JUNIOR, J. M. S.; LEÃO, L. R. S.; BRITO, M. V. H.; PA, T.C.B.C. et al. 
Efeitos do óleo de andiroba (Carapa guianensis) na função hepática de ratos submetidos à isquemia e reperfusão normotérmica do fígado. Revista do Colégio Brasileiro de Cirurgiões, v. 40, n. 6, p. 476-479, 2013. Disponível em: <http://dx.doi.org/10.1590/S0100-69912013000600010>. doi: 10.1590/S0100 69912013000600010

FARIAS, M. P. O; TEIXEIRA, W. C.; WANDERLEY, A. G.; ALVES, L. C.; FAUSTINO, M. A. G et al. Avaliação in vitro dos efeitos do óleo da semente de Carapa guianensis Aubl. Sobre larvas de nematóides gastrintestinais de caprinos e ovinos. Revista Brasileira de Plantas Medicinais, v.12, n. 2, p. 220-226, 2010. Disponível em: <https://doi.org/10.1590/S1516-05722010000200015>. doi: 10.1590/S151605722010000200015

FARIAS, M. P. O.; SOUSA, D. P.; ARRUDA, A. C.; WANDERLEY, A. G., TEIXEIRA, W.C. et al. Potencial acaricida do óleo de andiroba Carapa guianensis Aubl. sobre fêmeas adultas ingurgitadas de Anocentor nitens Neumann, 1897 e Rhipicephalus sanguineus Latreille, 1806. Arquivo Brasileiro de Medicina Veterinária e Zootecnia, v. 61 , n. 4, p. 877-882, 2009. Disponível em: <https://doi.org/10.1590/S0102-09352009000400015 >. doi: 10.1590/S010209352009000400015

FERNANDES, C. P. M.; MACHADO, C.; LOPES, T. V.; FILHO, N. C., BRETANHA, P. R. et al. Repellent Action of Carapa guianensis and Caesalpinia ferrea for flies species of Calliphoridae family. Ciência Rural, v. 46, n. 5, p. 867-870, 2016. Disponível em: <https://doi.org/10.1590/0103-8478cr20150727>. doi: 10.1590/01038478 cr20150727

FERRARI, M.; OLIVEIRA, M. S. C.; NAKANO, A. K. ROCHA FILHO, P. A. Determinação do fator de proteção solar (FPS) in vitro e in vivo de emulsões com óleo de andiroba (Carapa guianensis). Revista Brasileira de Farmacognosia, v. 17, n. 4, p. 626-630, 2007. Disponível em: <https://doi.org/10.1590/S0102695X2007000400023>. doi: 10.1590/S0102-695X2007000400023

HENRIQUES, M. G.; PENIDO, C. The therapeutic properties of Carapa guianensis. Current Pharmaceutical Design, V. 20, N. 6, p. 850-856, 2014. Disponível em: <https://doi.org/10.2174/13816128113199990048> $10.2174 / 13816128113199990048$

HIGUCHI, K.; MIYAKE, T.; OHMORI, S.; TANI, Y.; MINOURA, K. et al. Carapanosins A-C from seeds of andiroba (Carapa guianensis, Meliaceae) and their effects on Ipsactivated NO production. Molecules, v. 22, n. 3, p. 1-9, 2017a. Disponível em: <https://doi.org/10.3390/molecules22030502>. doi: 10.3390/molecules22030502

HIGUCHI, K.; TANI, Y.; KIKUCHI, T.; EM, Y.; YAMADA, T. et al. Guianolactones A and $\mathrm{B}$, two rearranged pentacyclic limonoids from the seeds of Carapa guianensis. Chemistry - An Asian Journal, v. 12, n. 23, p. 3000 - 3004, 2017b. Disponível em: <https://doi.org/10.1002/asia.201701298>. doi: 10.1002/asia.201701298 
INOUE T.; OHMORI, S.; KIKUCHI, T.; YAMADA, T.; TANAKA, R. Carapanosins d-f from the seeds of andiroba (Carapa guianensis, Meliaceae) and their effects on Ipsactivated no production. Molecules, v. 23, n. 7, p. 1-9, 2018. Disponível em: $<10.3390 /$ molecules23071778> doi: 10.3390/molecules23071778

JESUS, F. L. M.; ALMEIDA, F. B.; DUARTE, J. L.; OLIVEIRA, A. E. M. F. M.; CRUZ R. A.S. et al. Preparation of a nanoemulsion with Carapa guianensis Aublet (Meliaceae) oil by a low-energy/solvent-free method and evaluation of its preliminary residual larvicidal activity. Hindawi, v. 2017, n. 1, p. 1-8, 2017. Disponível em: <https://doi.org/10.1155/2017/6756793>. doi: 10.1155/2017/6756793

KLAUCK, V.; PAZINATO, R.; STEFANI, L. M.; SANTOS, R. C.; VAUCHER, R. A. et al. Insecticidal and repellent effects of tea tree and andiroba oils on flies associated with livestock. Medical and Veterinary Entomology, v, 28, n. 1, p. 33-39, 2014. Disponível em: <https://doi.org/10.1111/mve.12078>. doi: 10.1111/mve.12078

KLAUCK, V.; PAZINATO, R.; RADAVELLI, W. M.; VOLPATO, A.; STEFANI, L. M. et al. In vitro repellent effect of tea tree (Melaleuca alternifolia) and andiroba (Carapa guianensis) oils on Haemotobia irritans and Chrysomya megacephala flies. Tropical Biomedicine, v. 32, n. 1, p. 160-166, 2015. Disponível em: <https://doi.org/10.1111/mve.12078>. doi: 10.1111/mve.12078

LEMES, S. R.; CHAVES, D. A.; JÚNIOR, N. J. S.; CARNEIRO, C. C.; CHEN-CHEN, L. et al. Antigenotoxicity protection of Carapa guianensis oil against mitomycin $C$ and cyclophosphamide in mouse bone marrow. Anais da Academia Brasileira de Ciências, v. 89, n. $3, \quad$ p. 2043-2051, 2017. Disponível em: <https://doi.org/10.1590/0001-3765201720150797>. doi: 10.1590/00013765201720150797

MECCIA, G.; QUINTERO, P.; ROJAS, L. B.; USUBILLAGA, A.; VELASCO, J. et al. Chemical composition of the essential oil from the leaves of Carapa guianensis collected from Venezuelan Guayana and the antimicrobial activity of the oil and crude extracts. Natural Product Communications, v. 8, n. 11, 2013. Disponível em: <http://dx.doi.org/10.2174/13816128113199990048>. doi: $10.2174 / 13816128113199990048$

MIRANDA JÚNIOR, R. N. C.; DOLABELA, M. F.; SILVA, M. N.; POVOA, M. M.; MAIA, J. G. S. et al. Antiplasmodial activity of the andiroba (Carapa guianensis Aubl., Meliaceae) oil and its limonoid-rich fraction. Journal of Ethnopharmacology, v. 142, n. 3 , p. 679-683, 2012. Disponível em: <http://dx.doi.org/ 10.1016/j.jep.2012.05.037>. doi: 10.1016/j.jep.2012.05.037

MOYA, R., RODRíGUEZ-ZÚÑIGA, A.; VEGA-BAUDRIT, J.; ÁLVAREZ, A. Effects of adding nano-clay (montmorillonite) on performance of polyvinyl acetate (PVAc) and urea-formaldehyde (UF) adhesives in Carapa guianensis, a tropical species. International Journal of Adhesion \& Adhesives, v. 59, n. 1, p. 62-70, 2015. Disponível em: <https://doi.org/10.1016/j.ijadhadh.2015.02.004>. doi: 10.1016/j.ijadhadh.2015.02.004 
NARDI, M.; GUEDES, A. C. L.; CUNHA, H. F. A.; GUEDES, M. C.; MUSTIN, K. et al. Artisanal extraction and traditional knowledge associated with medicinal use of crabwood oil (Carapa guianensis Aublet.) in a peri-urban várzea environment in the Amazon estuary. Hindawi, v. 2016, n. 1, p. 1-12, 2016. Disponível em: <https://doi.org/ 10.1155/2016/5828021>. doi: 10.1155/2016/5828021

NAYAK, B. S.; KANHAI.; J.; MILNE, D. M.; SWANSTON, W. H.; MAYERS, S. et al. Investigation of the wound healing activity of Carapa guianensis I. (meliaceae) bark extract in rats using excision, incision, and dead space wound models. Journal of Medicinal Food, v. 13, n. 5, p. 1141-1146, 2010. Disponível em: < http://dx.doi.org/10.1089/jmf.2009.0214.>. doi: 10.1089/jmf.2009.0214.

OLIVEIRA, I. D. S. D. S.; MORAGAS, C. J. T.; CHAGAS, M. D. S. D. S.; BEHRENS M. D.; CALABRESE, K. D. S. et al. Carapa guianensis Aublet (Andiroba) seed oil: chemical composition and antileishmanial activity of limonoid-rich fractions. BioMed Research International, p. $1-10$ 2018. Disponível em: <https://doi.org/10.1155/2018/5032816>. doi: 10.1155/2018/5032816

PAIXÃO, S. S. R. M.; FASCINELI, M. L.; ROLL, M. M.; LONGO, J. P. F.; AZEVEDO, R. B. et al. The lipidome, genotoxicity, hematotoxicity and antioxidant properties of andiroba oil from the Brazilian Amazon. Genetics and Molecular Biology, v. 39, n. 2, p. 248-256, 2016. Disponível em: <http://dx.doi.org/10.1590/1678-4685-GMB2015-0098>. doi: 10.1590/1678-4685-GMB-2015-0098

PARREIRA, D. S.; ALCÂNTARA-DE-CRUZ, R.; LEITE, G. L. D.; RAMALHO, F. S.; ZANUNCIO, J. C. et al. Quantifying the harmful potential of ten essential oils on immature Trichogramma pretiosum stages. Chemosphere, v. 199, p. 670-675, 2018. Disponível em: <https://doi.org/10.1016/j.chemosphere.2018.02.083>. doi: 10.1016/j.chemosphere.2018.02.083

PENIDO, C.; COSTA, K. A.; PENNAFORTE, R. J.; COSTA, M. F. S.; PEREIRA, J. F. G. et al. Anti-allergic effects of natural tetranortriterpenoids isolated from Carapa guianensis Aublet on allergen-induced vascular permeability and hyperalgesia. Inflammation Research, v. 54, n. 7, p. 295-303, 2005. Disponível em: <http://dx.doi.org/10.1007/s00011-005-1357-6>. doi: 10.1007/s00011-005-1357-6

PENIDO, C.; CHAGAS, M. S. S.; RODRIGUES, C. A. B.; PEREIRA, J. F. G.; HENRIQUES, M. G. M. O. et al. Antiinflammatory effects of natural tetranortriterpenoids isolated from Carapa guianensis Aublet on zymosan-induced arthritis in mice. Inflammation Research, v. 55, n. 11, p. 457-464, 2006. Disponível em: <http://dx.doi.org/10.1007/s00011-006-5161-8>. doi: 10.1007/s00011-006-51618

PEREIRA, T. B.; SILVA, L. F. R. E.; AMORIM, R. C. N.; MELO, M. R. S.; SOUZA, R. C. Z. et al. In vitro and in vivo anti-malarial activity of limonoids isolated from the residual seed biomass from Carapa guianensis (andiroba) oil production. Malaria Journal. v. 13, n. 317, p. 1-8, 2014. Disponível em: <https://doi.org/10.1186/14752875-13-317>. doi: 10.1186/1475-2875-13-317 
PROPHIRO, J. S.; SILVA, M. A. N.; KANIS, L. A.; SILVA, B. M.; LUNA, J. E. D. et al. Evaluation of time toxicity, residual effect, and growth-inhibiting property of Carapa guianensis and Copaifera sp. in Aedes aegypti. Parasitology Research, v. 110, n. 2, p. 713-719, 2012. Disponível em: <http://dx.doi.org/10.1007/s00436-011-2547-5 >. doi: $10.1007 / \mathrm{s} 00436-011-2547-5$

PROPHIRO, J. S.; SILVA, M. A. N.; KANIS, L. A.; ROCHA, L. C. B. P.; LUNA, J. E. D. et al. First report on susceptibility of wild Aedes aegypti (Diptera: Culicidae) using Carapa guianensis (Meliaceae) and Copaifera sp. (Leguminosae). Parasitology Research, v. 110, n. 2, p. 699-705, 2012. Disponível em: <http://dx.doi.org/10.1007/s00436-011-2545-7>. doi:10.1007/s00436-011-2545-7

ROMA, G. C.; MATHIAS, M. I. C.; NUNES, P. H.; REMÉDIO, R. N.; BECHARA, G. $\mathrm{H}$. et al. Effects of andiroba (Carapa guianensis) oil in ticks: Ultrastructural analysis of the synganglion of Rhipicephalus sanguineus (Latreille, 1806) (Acari: Ixodidae). Acta Tropica, v. 141, n. 1, p. 7-15, 2015. Disponível em: <http://dx.doi.org/10.1590/S0100-204X2007000900011>. doi: 10.1016/j.actatropica.2014.06.018

ROMA, G. C.; MATHIAS, M. I. C.; FARIA, A. U.; OLIVEIRA, P. R.; FURQUIM, K. C. $S$. et al. Morphological and cytochemical changes in synganglion of Rhipicephalus sanguineus (Latreille, 1806) (Acari: Ixodidae) female ticks from exposure of andiroba oil (Carapa guianensis). Microscopy Research and Technique, v. 76, n. 7, p. 687696, 2013. Disponível em: <http://dx.doi.org/10.1590/S0100-204X2007000900011>. doi: 10.1590/S0100-204X2007000900011

ROSA, J. M.; BOFF, M. I. C.; GONÇALVES, P. A.; BOFF, P.; NUNES, M. Z. Andiroba oil (Carapa guianensis Aubl) in the capture of the fruit fly (Anastrepha fraterculus Wiedemann) in Feijoa (Acca sellowiana (Berg) Burret). IDESIA. v. 31, n. 3, p. 97-101, 2013. Disponível em: <http://dx.doi.org/10.4067/S071834292013000300013>. doi: 10.4067/S0718-34292013000300013

SAKAMOTO, A.; TANAKA, Y.; YAMADA, T.; KIKUCHI, T.; MURAOKA, O. et al. Andirolides $\mathrm{W}-\mathrm{Y}$ fromthe flower oil of andiroba (Carapa guianensis, Meliaceae). Fitoterapia, v. 100, n. 1, p. 81-87, 2015.

SANTOS, M. N., CUNHA, H.F.A., LIRA-GUEDES, A.C., GOMES, S.C.P, GUEDES, M.G. Saberes tradicionais em uma unidade de conservação localizada em ambiente periurbano de várzea: etnobiologia da andirobeira (Carapa guianensis Aublet), Boletim do Museu Paraense Emílio Goeldi. Ciências Humanas, v. 9, n. 1, p. 93108, $2014 . \quad$ Disponível em: $<$ https://ainfo.cnptia.embrapa.br/digital/bitstream/item/102441/1/CPAF-AP-2014Saberes-tradicionais.pdf $>$.

SANTOS, O. J.; MALAFAIA, O.; FILHO, J. M. R.; CZECZKO, N. G.; SANTOS, R. H. $P$. et al. Efeito de Schinus terebinthifolius raddi (aroeira) e Carapa guianensis aublet (andiroba) na cicatrização de gastrorrafias. Arquivo Brasileiro de Cirurgia Digestiva, v. 2 , n. 1 , p. 84-91, 2013. Disponível em: <http://dx.doi.org/10.1590/S0102-67202013000200003>. doi: 10.1590/S010267202013000200003 
SANTOS, R. S.; PELLICCIOTTI, A. S. Ocorrência de Hypsipyla ferrealis Hampson (Lepidoptera: Pyralidae) em Andiroba no Estado do Acre. Ciência Florestal, v. 26, n. 3, p. 995-998, jul.-set., 2016. Disponível em: <http://dx.doi.org/10.5902/1980509824228>. doi: 10.5902/1980509824228

SILVA, C. E. S.; SANTOS, O. J.; FILHO, J. M. R.; TABUSHI, F. I.; KUME, M. H. et al. Efeito da Carapa guianensis Aublet (Andiroba) e Orbignya phalerata (Babaçu) na cicatrização de colorrafias em ratos. Revista do Colégio Brasileiro de Cirurgiões, v. 42, n. 6, p. 399-406, 2015. Disponível em: <http://dx.doi.org/10.1590/010069912015006009>. doi: 10.1590/0100-69912015006009

SILVA, F. R. P.; ALMEIDA, S. S. M. S. Análise fitoquímica e microbiológica da atividade do extrato bruto etanólico da Andiroba, Carapa guianensis Aubl. Biota Amazônia, Macapá, v. 4, n. 4, p. 10-14, 2014. Disponível em: <http://dx.doi.org/10.18561/2179-5746/biotaamazonia.v4n4p10-14>. doi: 10.18561/2179-5746/biotaamazonia.v4n4p10-14

SILVA, O. S.; PROPHIRO, J. S.; NOGARED, J. C.; KANIS, L.; EMERICK, S. et al. Larvicidal effect of andiroba oil, Carapa guianensis (meliaceae), against Aedes aegypti. Journal of the American Mosquito Control Association, v. 22, n. 4, p. 699-701, 2006. Disponível em: <http://dx.doi.org/ 10.2987/8756971X(2006)22[699:LEOAOC]2.0.CO;2>. 1oi: 10.2987/8756971X(2006)22[699:LEOAOC]2.0.CO;2

SOUZA, B. A. A.; BRAGA, L. A.; LOPES, L. R. O.; JUNIOR, R. F. G. R.; NASCIMENTO, L. N. S. et al. Effects of andiroba oil (Carapa Guianensis) on wound healing in alloxan-diabetic rats. International Archives of Medicine, v. 10, n. 263, p. 1-6, 2017. Disponível em: <http://dx.doi.org/10.3823/2533 >. doi: 10.3823/2533

TONINI, H.; OLIVEIRA JÚNIOR, M. M. C.; SCHWENGBER, D. Crescimento de espécies nativas da amazônia submetidas ao plantio no Estado de Roraima. Ciência Florestal, v. 18, n. 2, p. 151-158, 2008. Disponível em: <https://periodicos.ufsm.br/cienciaflorestal/article/view/453/350>.

TORRES, S. M.; CRUZ, N. L. N.; ROLIM, V. P. M.; CAVALCANTI, M. I. A.; ALVES, L. C. et al. Mortalidade acumulativa de larvas de Aedes aegypti tratadas com compostos. Revista de Saúde Pública, v. 48, n. 3, p. 445-450, 2014. Disponível em: <http://dx.doi.org/10.1590/S0034-8910.2014048005022>. doi: 10.1590/S00348910.2014048005022

WANZELER, A. M. V.; JÚNIOR, S.M.A.; GOMES, J.T.; GOUVEIA, E.H.H.; HENRIQUE H.Y.B. et al. Therapeutic effect of andiroba oil (Carapa guianensis Aubl.) against oral mucositis: an experimental study in golden Syrian hamsters. Clinical Oral Investigation, v. 22, n. 5, p. 2069-2079, 2018. Disponível em: <http://dx.doi.org/10.1007/s00784-017-2300-2>. doi: 10.1007/s00784-017-2300-2 